\title{
Rising interdisciplinary collaborations refine our understanding of autisms and give hope to more personalized solutions
}

\begin{abstract}
Autism is heterogeneous, complex and arguably a condition of many conditions. Both the number of researchers and the number of research collaborations in the field of autism have been growing at unprecedented rates. Interdisciplinary collaborations have increased more than eightfold since the year 2000. In fact, most - if not all areas of autism research are starting to converge, and these convergences are leading not only to a richer research network but also to a causal network for autism. This network can, and likely will, decode the many forms of autism into its various subcomponents, enabling increasingly more personalized approaches for both the detection and treatment of those different forms of autism.
\end{abstract}

\section{Keywords: autism spectrum disorders $\bullet$ collaborative research • developmental disorders - interdisciplinary research • personalized medicine}

The prevalence of autism, a spectrum of neuropsychiatric disorders characterized by deficits in social interaction and communication as well as restricted and repetitive behaviors, has been on a steady rise in the USA, most recently reported by the $\mathrm{CDC}$ to affect as many as one in 68 individuals, and one in 42 boys [1]. With the increased volume of research fueled by increased awareness, it has become apparent that autism spectrum disorders (ASDs) are marked by a large degree of genetic and phenotypic heterogeneity, and its etiology cannot be attributed to a single cause, but rather a combination of several factors [2]. The field generally agrees that there are many forms of autism. However, just how many forms remains unknown, and currently whatever forms can be identified are defined solely by behavioral characteristics, many of which overlap with other developmental disorders such as attention deficit hyperactivity disorder and obsessive-compulsive disorder [3]. Currently, there is no approach that allows the various forms of autism to be easily and accurately disentangled from one another.

The complex and heterogeneous nature of autism has necessitated a transition away from traditional methods toward a more cooperative research landscape, encouraging investigators to collaborate with individuals outside their classically defined disciplines. Recent studies have shown that collaborative [4] and interdisciplinary [5] research may lead to faster, more impactful discoveries. These large, multidisciplinary collaborations are working to parse the current spectrum of autism into its many subcomponents, carving the path toward an increasingly more personalized diagnostic and therapeutic process.

In the present work, we implement intelligent data mining strategies to construct the autism bibliome - a complete database of published research studies related to autism from the last 15 years. Through this bibliometric analysis we are able to compute the rise in research efforts focused on autism, as well as identify the key investigators that have made the most impactful discoveries in this field and quantify the collaborations between these top players. We further assess the nature of these research collaborations to determine if, and at what capacity, various subdisciplines have worked together to begin to decode the complexity of ASD.

\section{Marlena Duda'}

\& Dennis P Wall*,1

'Department of Pediatrics \& Psychiatry (by courtesy), Division of Systems

Medicine, Stanford University, Stanford, CA 94305, USA

*Author for correspondence:

Tel.: +1 6174323894

Fax: +1 6174320693

dpwall@stanford.edu
Future fsg 
Through the use of author-specific keyword ontologies we were able to quantify the proportion of all collaborations that occurred between researchers in different disciplines.

\section{Methods}

\section{Search strategy}

We mined the PubMed database to develop the autism bibliome - an authoritative set of publications relating to autism spectrum disorders from the years 2000 to 2014 (publication history obtained May 2014). These results were filtered so the final dataset consisted only of publications that were explicitly affiliated with institutions in the USA. Publication dates as well as author and keyword information were obtained via PubMed Entrez Utilities [6]. To address authors who have used multiple aliases, we collapsed publication lists of both aliases when there was only one possible pairing of initials (i.e., collapse J Smith and JA Smith, if no other JX Smith). Since we do not discriminate between first, last and middle authors in our database, we decided to refine our analyses to only authors that have contributed three or more publications to the bibliome. Our final filtered data repository consisted of 13,939 publications and 9,492 unique authors. Figure 1 provides a visual representation of our data mining methods.

As a way to characterize authors by their research interests, we analyzed the total aggregated set of keywords from the author's publication list. We used fuzzy string matching in Python to pair similar keyword terms (i.e., development and developmental), then ranked the terms in this keyword ontology by frequency of appearance. Each author's discipline was defined by their top five keywords, and natural language processing algorithms were used to assess the

\section{O Autism OR Asperger OR developmental delay Publication date Affiliation From 1 January $2000 \quad$ Includes 'USA'}
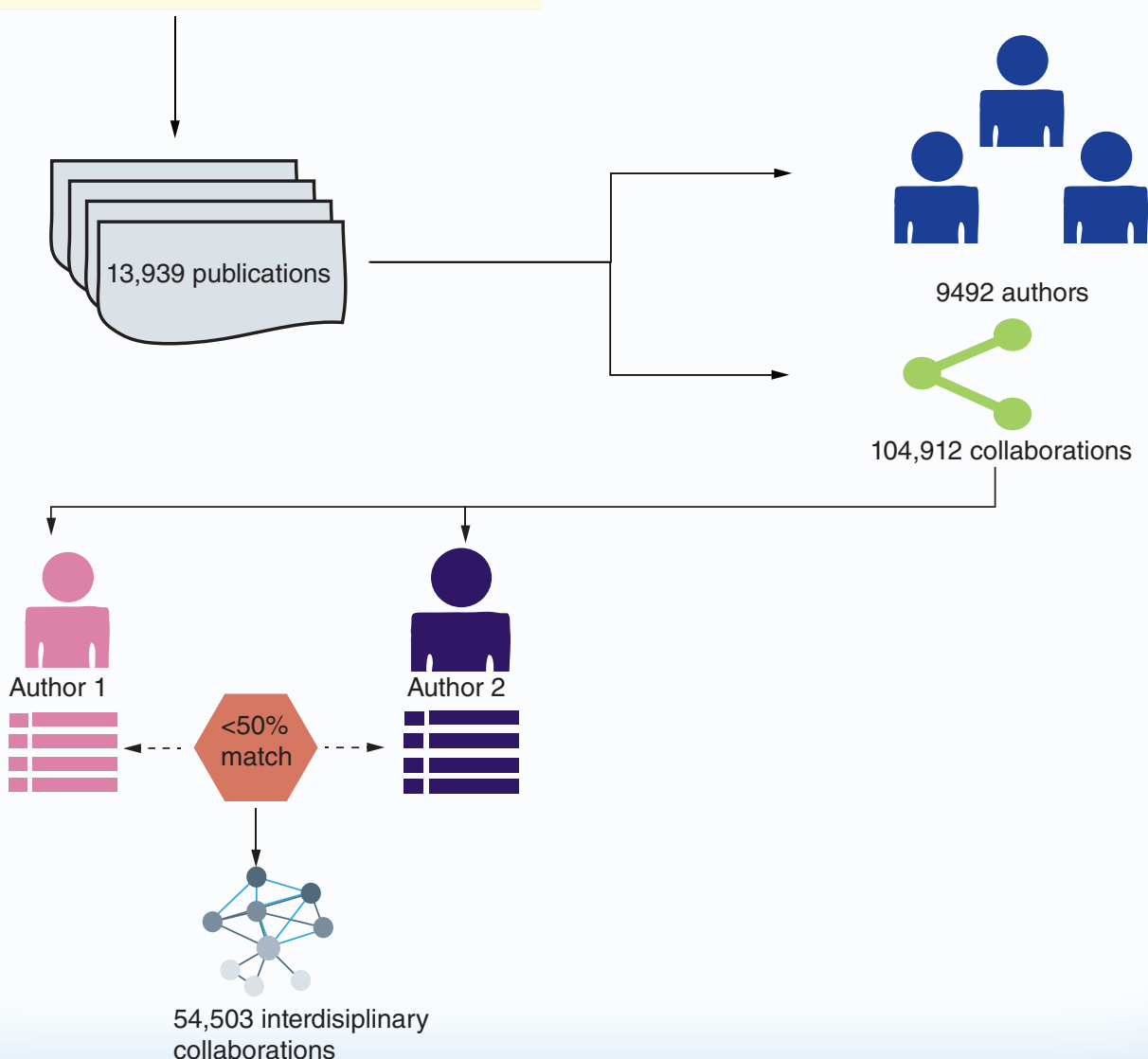

Figure 1. Flow chart of data mining methods. Our PubMed query yielded 13,939 publications, 9492 authors and 104,912 collaborations. Author's disciplines were defined by their five most frequently used keywords and co-author pairs were categorized as single or inter-disciplinary based on a comparison of their ranked keyword lists, resulting in 54,503 interdisciplinary collaborations. 
(dis) similarity of disciplines between co-author pairs. Co-authors with disciplines that were $50 \%$ or more dissimilar were classified as interdisciplinary, and those that failed to meet this threshold were considered single disciplinary. For our purposes, the term 'collaboration' represented a single instance of coauthorship, meaning a single co-author pair may have two or more collaborations, and a publication with four authors would contribute six collaborations to the yearly total, one for each co-author pair.

\section{Statistical analysis}

In order to determine the effect of interdisciplinary collaborations on impact of research, we used the 2013 Thompson Reuters Journal Citation Reports [7] to find journal impact factor (IF) data, which were available for a subset of 8,488 publications. We then computed the average IF for both interdisciplinary and single disciplinary collaborations. Using $\mathrm{R}$, we ran a Student's $t$-test to compare the groups and investigate whether or not significant difference in impact existed between them.

\section{Results}

\section{Growth surrounding autism}

Our mining of the autism bibliome yielded 13,939 unique research studies between the years 2000 and 2014. In total, this set of publications represented 31,804 unique authors and 69,305 coauthor pairs.
We limited our analyses to include only authors with three or more contributions to the bibliome. This subset of 9,492 authors has contributed to 104,897 unique collaborations, defined as a single instance of co-authorship between two individuals.

Over this 15-year time frame, we calculated a significant rise in both the number of unique research studies and the number of researchers overall. At the peak of activity in 2013, there was a $194 \%$ increase in publications and a $241 \%$ increase in top publishing authors as compared with the year 2000 (Table 1). Overall, we saw a steadily increasing trend across all years for both authors and publications from 2000 to 2013 (Figure 2A).

Not only did we find a significant increase in the amount of research being done in autism, we also discovered a shift in the way in which this research was being conducted. Our data showed as much as a $645 \%$ increase in all collaborations and an even greater increase $-760 \%$ - in interdisciplinary collaborations from 2000 to 2012 (Table 1 \& Figure 2B). In fact, of the 104,897 total collaborations, 54,503 (52\%) were classified as interdisciplinary connections. To explore the interdisciplinary nature of the autism research network even further, we calculated an interdisciplinary score (IS) for each author per year, defined simply as the percentage of all the author's interdisciplinary collaborations. The average author's IS increased nearly $20 \%$, from 0.429 in 2000 to 0.622 in 2014, and the number

\section{Table 1. Yearly breakdown of all metrics.}

\begin{tabular}{|c|c|c|c|c|c|}
\hline Year & Publications & Authors & $\begin{array}{l}\text { Co-author } \\
\text { pairs }\end{array}$ & Collaborations $^{\dagger}$ & $\begin{array}{l}\text { Interdisciplinary } \\
\text { collaborations }\end{array}$ \\
\hline 2000 & 458 & 914 & 1743 & 2034 & 915 \\
\hline 2001 & 485 & 1024 & 2291 & 2568 & 1148 \\
\hline 2002 & 526 & 1166 & 2664 & 3002 & 1335 \\
\hline 2003 & 521 & 1161 & 2134 & 2255 & 942 \\
\hline 2004 & 608 & 1418 & 3139 & 3580 & 1555 \\
\hline 2005 & 651 & 1566 & 3924 & 4426 & 1860 \\
\hline 2006 & 731 & 1773 & 4515 & 5128 & 2215 \\
\hline 2007 & 780 & 1933 & 4721 & 5211 & 2235 \\
\hline 2008 & 928 & 2236 & 6902 & 7703 & 3582 \\
\hline 2009 & 905 & 2319 & 9933 & 11,627 & 6163 \\
\hline 2010 & 1060 & 2592 & 8096 & 9151 & 4407 \\
\hline 2011 & 1161 & 2930 & 10,355 & 11,409 & 5443 \\
\hline 2012 & 1229 & 3158 & 13,391 & 15,155 & 7866 \\
\hline 2013 & 1348 & 3113 & 11,804 & 13,518 & 7418 \\
\hline 2014 & 524 & 1601 & 7709 & 8130 & 7419 \\
\hline
\end{tabular}


(A)

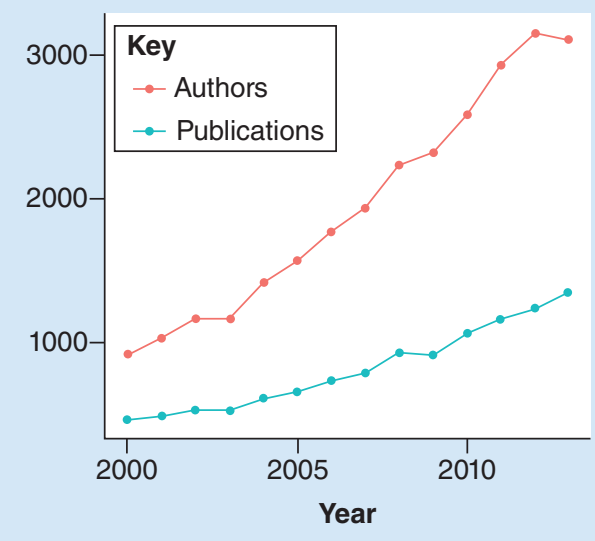

(B)

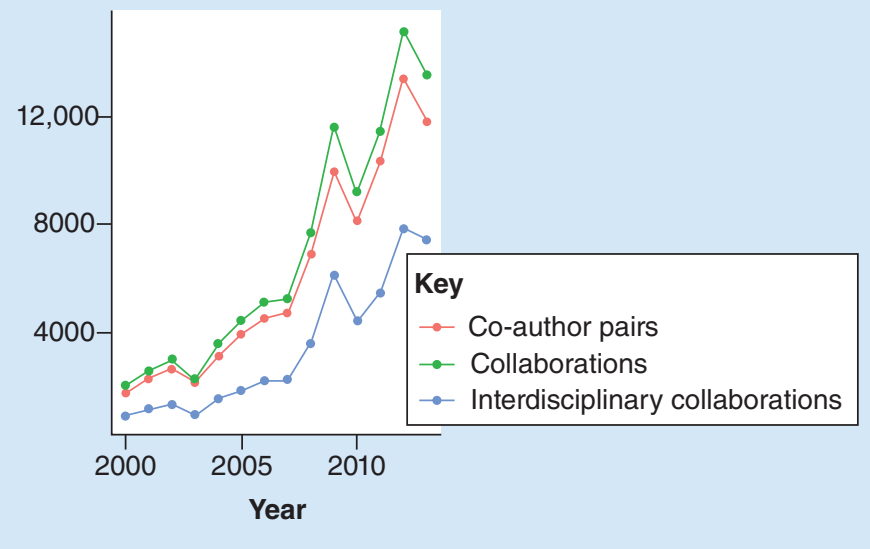

Figure 2. Publication and collaboration growth in autism research. Publication and collaboration trends in autism from 2000 to 2013. From these graphs it is apparent that not only is the rate of research in autism rising (A), but also that the field is becoming more collaborative as a whole, and that the nature of these collaborations is becoming increasingly inter-disciplinary (B).

For color images please see online at: www.futuremedicine.com/doi/full/10.2217/PME.15.8

of authors participating in interdisciplinary research at any level rose from 67.94 to $84.38 \%$ within that same timeframe. This represents a growing network of interdisciplinary work (Figure 3), suggesting that the field as a whole is coming together around some of the major unsolved questions in autism to identify markers and clinical targets that will likely lead to more personalized approaches.

Given that interdisciplinary research in autism has risen dramatically, the next question is whether this has elevated the impact and clinical importance of the research findings themselves. To address this question, we obtained the 2013 Thompson Reuters Journal Citation Reports [7], and matched journal IF data to 8,488 of our publications (IF not available for the remainder). Using this subset, we computed the average IF for both single disciplinary and interdisciplinary collaborations, and using a student's $t$-test, found that interdisciplinary collaborations had a significantly higher average IF (10.14) as compared with single disciplinary collaborations (7.40; $\mathrm{p}<<0.05)$.

\section{Discussion}

Impact of cross-disciplinary efforts

Catalyzed by an increasing consensus that autism is a constellation of disorders, the nature of autism research has moved in dramatic and decisive fashion from isolated research in specific disciplines to highly interdisciplinary collaborations that reach from clinical psychiatry through to machine learning and computational approaches in 'Big Data'. These interdisciplinary collaborations have resulted in growing datasets that enable high-powered statistical analyses as well as innovative multi-perspective approaches that, when paired, can provide valuable insight to the existing problems in the etiology, diagnosis and treatment of autism that will likely lead to a more accurate and individualized clinical process.

\section{Etiology}

Due to its strong heritability despite large genetic heterogeneity, the molecular etiology of autism has been of large interest. Collaborations among cell biologists, systems biologists and researchers whose work has focused more on cancer, have pinpointed via the convergence of autism risk factors the likely importance of protein isoform interactions in the onset of autism. By creating the largest experimental protein isoform network to date (the Autism Spliceform Interaction Network), they have identified 20 new protein players in ASD, most of which are the nonreference isoform [8]. Collaborations among psychologists, geneticists and molecular biologists have discovered that the additive effects of multiple common variants contribute the majority of total genetic ASD risk, especially in multiplex families $[9,10]$, and a group of geneticists, neuroscientists and statisticians have collaborated to develop a statistical model to better interpret the significance of de novo mutations, based on gene-specific probabilities for five different types of mutations [11]. This model was then applied to 1078 trios, and the results showed no significant de novo ASD signals in cases with IQ $>100$, suggesting de novo mutations play a role in fundamental neurodevelopmental processes in autism. With over 6000 autism-related exomes sequenced to date [12-17], these innovative approaches and others like them are, and 
will continue, bringing us closer to understanding the molecular basis of autism, in turn providing targets for the development of personalized clinical tools for diagnosis and treatment of these disorders.

Although there is strong evidence for a genetic predisposition to autism, research has shown that the contribution of DNA alone is not enough to fully explain autism, and the contribution of environmental factors cannot be discounted. Recent interdisciplinary work involving biomedical informatics and epidemiology identified a strong correlation between environment, measured via the rate of congenital reproductive malformations in males, and autism incidence. While more work to disentangle the environmental cause of male reproductive malformation is needed, this is a critical step forward in finding environmental links to certain forms of autism [18].

\section{Diagnosis}

Currently, autism is diagnosed through behavioral evaluation with standard clinical instruments, such as the Autism Diagnostic Observation Schedule (ADOS) [19] and the Autism Diagnostic Interview Revised (ADI-R) [20]. Although these tools perform with high diagnostic accuracy, there remains a strong need for and a sharp focus in the field on the identification of biomarkers that can increase detection accuracy and also provide personalized assessments and treatments.

One approach to the biomarker question is the use of brain imaging to find structural differences in specific regions of the brain between ASD and non-ASD individuals. By applying multivariate pattern analysis to MRI neuroimaging data, researchers developed a classifier that performed with up to $90 \%$ accuracy based on gray matter [21], and a collaboration between child psychologists, geneticists and neuroimaging specialists found promising differences between the white matter fiber tract of ASD cases and controls that could be used to distinguish autism risk in infants as young as 6 months of age [22]. Another approach, integrating computational biology and neuroscience, has applied machine learning algorithms to infant resting EEG data to create a classifier that performed with $>80 \%$ diagnostic accuracy [23]. In light of the newly developed Autism Brain Imaging Data Exchange open access database containing over 1000 brain images [24] there are many opportunities to continue with validation of this noninvasive approach for the detection of autism.

Another key aim of autism biomarker research is focused on pinpointing the molecular signature of the disorder. There have been promising attempts to construct diagnostic classifiers based on genetic markers, specifically SNPs; however, the accuracy was found to vary among different ethnic groups [25]. Researchers have also explored the efficacy of using gene-expression abnormalities as biomarkers for ASD. Glatt et al. utilized microarray expression profiling of blood samples from infants and toddlers to develop a 48-probe classifier that was able to distinguish ASD from nonspectrum with $91 \%$ accuracy [26]. A similar, but larger study using 170 ASD cases and 115 controls developed a 55-gene prediction model for that shows promise for use as an autism classification tool [27] and is currently trialing at 20 clinical locations nationwide [28].

Though there is a great deal of promising evidence for the use of molecular signatures and neuroimaging techniques as diagnostic biomarkers for autism, these modalities require extensive research before they can be implemented clinically. Recently, there have been efforts to accelerate the currently accepted behavioral diagnostic process in hopes of achieving earlier and more rapid detection of autism, without sacrificing accuracy. By applying machine learning methodologies to the gold standard ADOS and ADI-R diagnostic exams, an interdisciplinary group of clinicians and bioinformaticians were able to develop two short classification tools for detecting autism that perform with $99 \%$ accuracy, while decreasing the length of the original exam by as much as $84 \%[29,30]$ and continues to validate with high accuracy, even when tested against newly revised scoring algorithms [31]. This screening modality could be of significant value as a clinical triaging tool, and also has the potential to be implemented in a mobilized online platform through short video analysis [32] and electronic parent questionnaires.

\section{Therapies}

Since the etiology of autism has yet to be fully understood, therapies are currently limited to behavioral treatments. Common behavioral therapies that are implemented today include Applied Behavioral Analysis (ABA) [33] and Pivotal Response Treatment [34,35], as well as the Early Start Denver Model [36]. Recently, there has been a push to incorporate advanced technologies into therapeutic tools for children with an autism diagnosis. There are currently over 500 Android and iPad apps that have been developed for children with autism, meant to aid in development of social and function skills, and some even implement parts of ABA [37]. A group of mathematicians, child psychologists and bioinformaticians is leveraging the eye-tracking capabilities of Google Glass as both a research tool to understand how children with autism see the world around them, and as a therapeutic to improve social 
(A) 2000

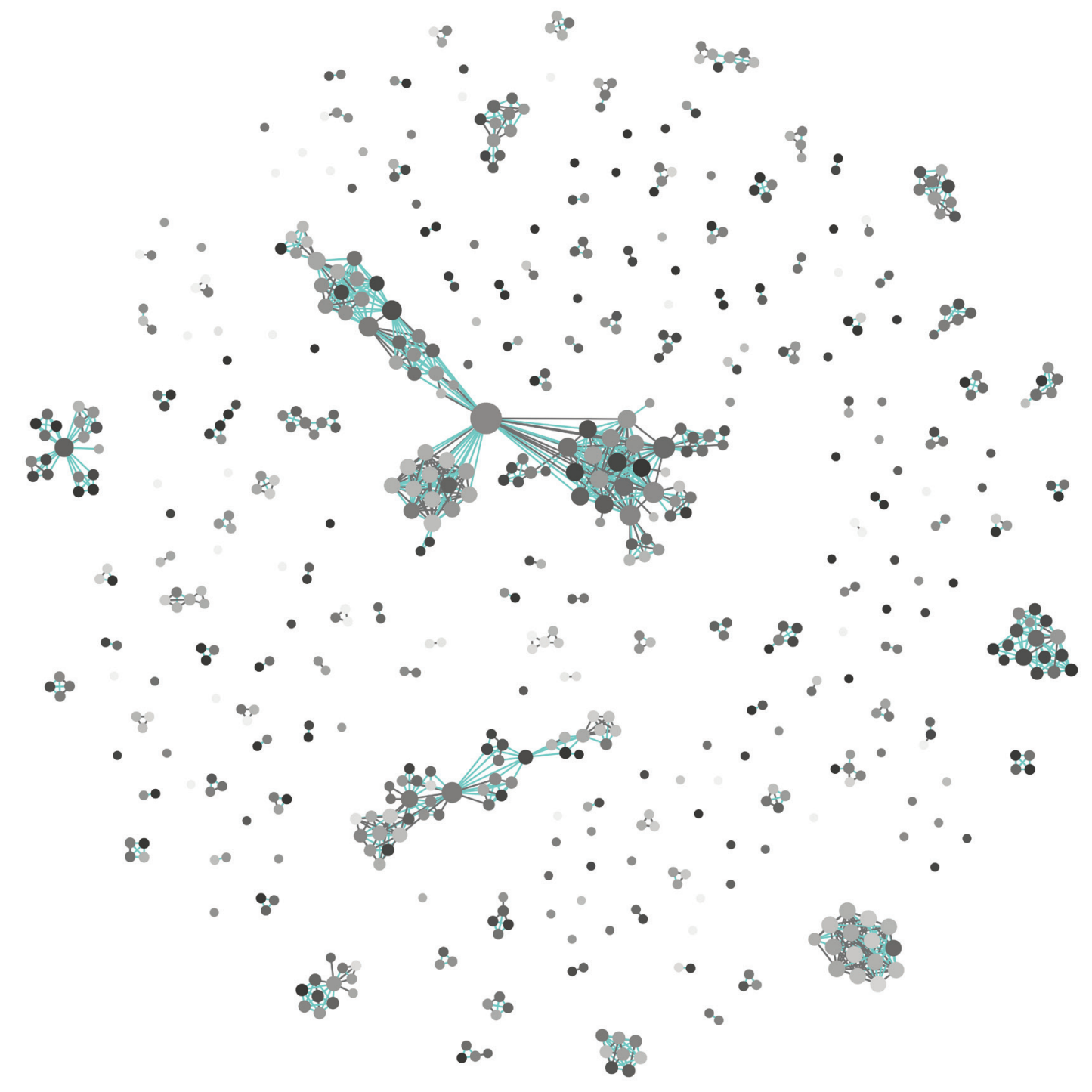

Interdisciplinary score

$\begin{array}{ll}0.0 & 1.0\end{array}$

Multidisciplinary connection

- Single-disciplinary connection 
(B) 2005

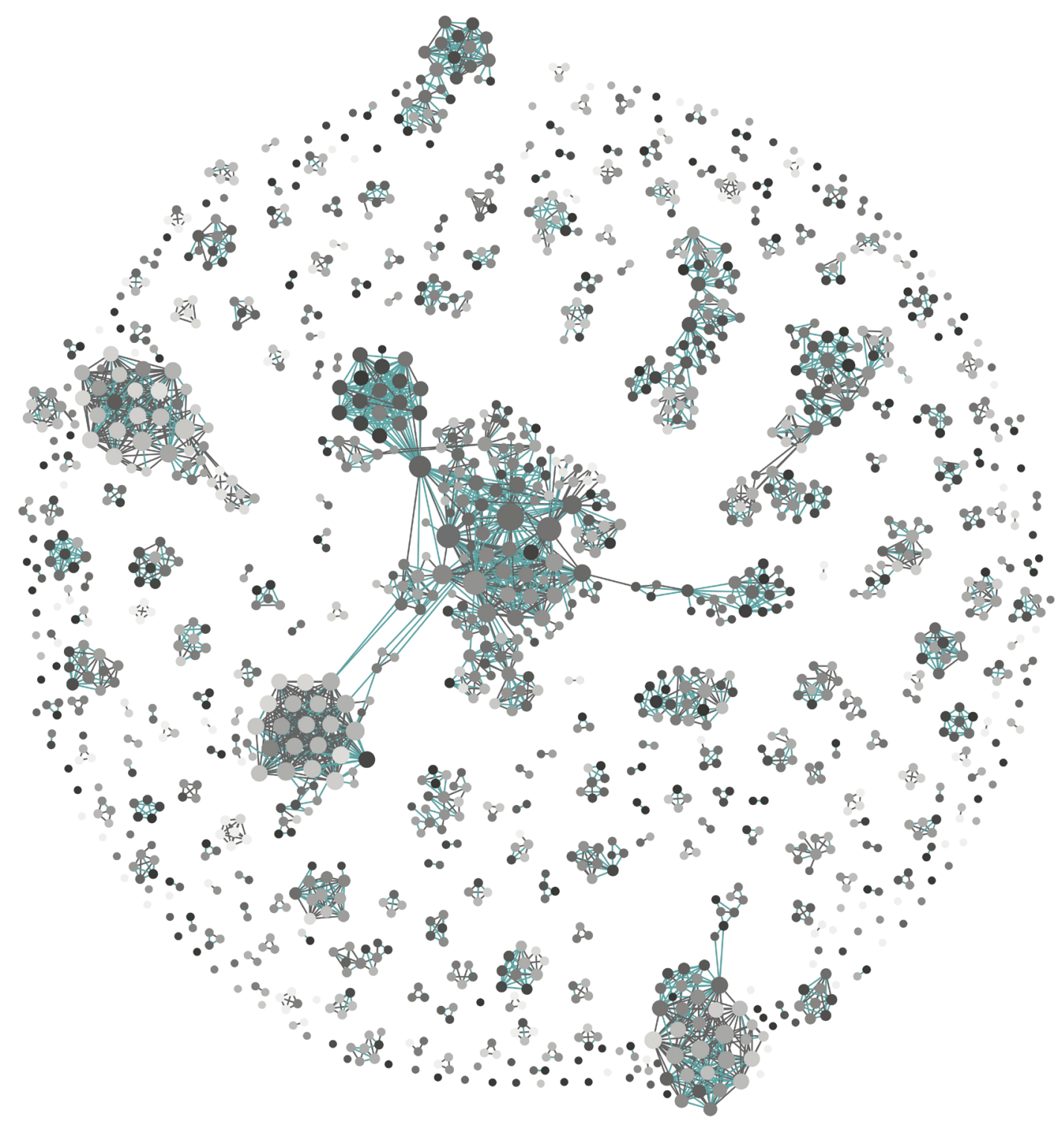

Interdisciplinary score

0.0

1.0

Multidisciplinary connection

Single-disciplinary connection 
(C) 2013

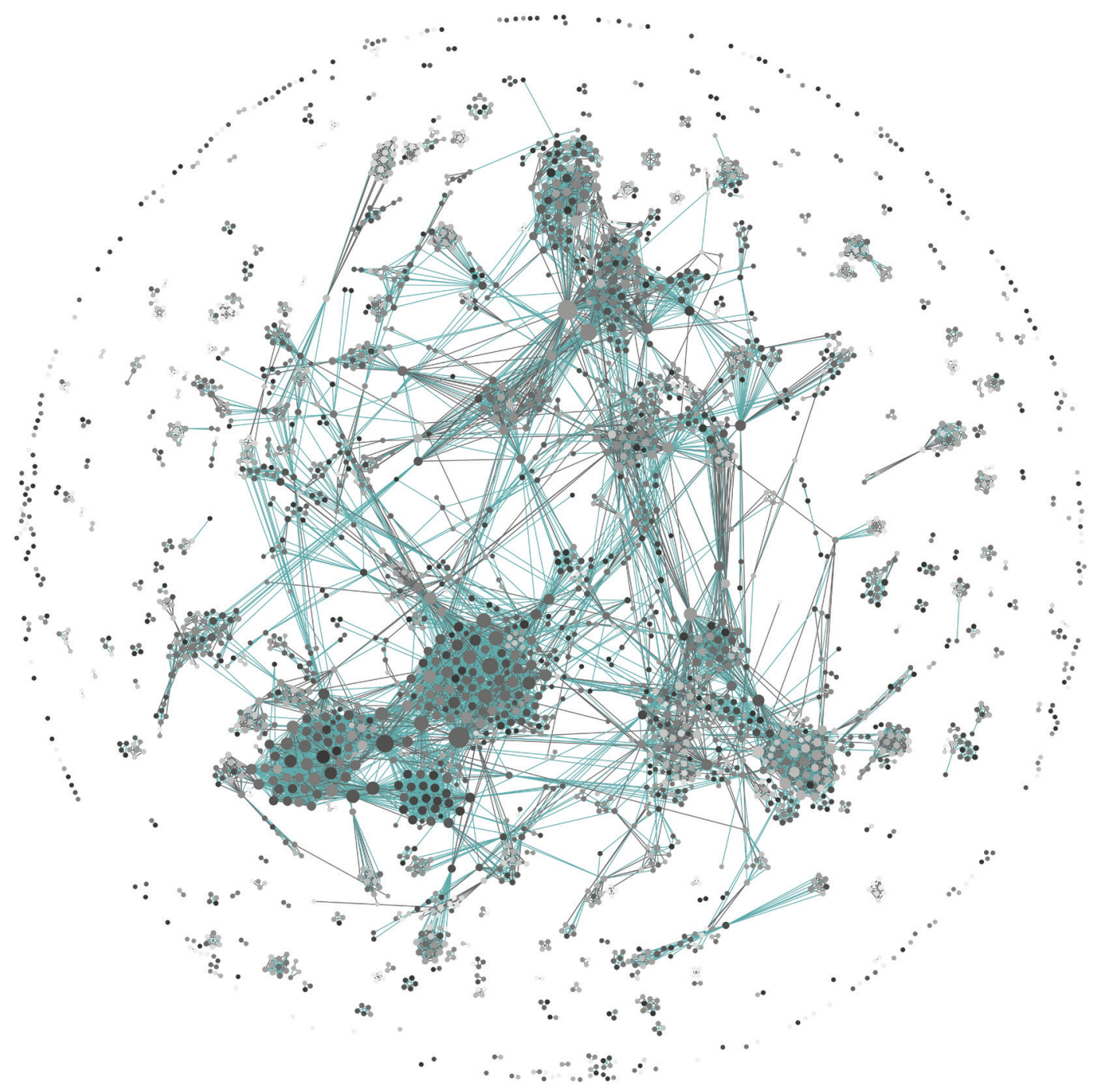

Interdisciplinary score

0.0

- Single-disciplinary connection 
Figure 3. Evolution of the autism research network from (A) 2000 to (B) 2005 to (C) 2013 (see pages 364 for [A], 365 for $[B]$ and 366 for $[C]$ ). Authors are represented as nodes, with node size corresponding to the degree of connectivity within the network and node color denoting the author's interdisciplinary score. Collaborations are represented as edges, with interdisciplinary collaborations highlighted in blue. Not only has this network grown in size, but it has also become more interconnected, specifically between authors from different disciplines.

interaction for children by helping them recognize and respond to social cues in real time [38]. A similar study utilized facial recognition software to develop a training program for individuals with ASD. They found that children who completed this training have improved facial processing, at both the behavioral and electrophysiological level, suggesting therapeutic validity for this training system [39]. As we gain insight into the molecular basis of autism, though, there are many other avenues of therapy to pursue, including drug [40] and stem cell therapies [41]. These kinds of treatments are advantageous due to their ability to be tailored to each patient and because they target the biological basis of the disease rather than just the symptoms, however more extensive research is required before they can be clinically implemented.

\section{Conclusion}

Over the last 15 years autism-related research efforts have increased threefold, involving over 9400 researchers and yielding more than 13,900 publications. Interdisciplinary collaborations have grown at an even faster pace $-84 \%$ of all collaborations in 2014 were between authors in different disciplines. This driving force in interdisciplinary research has resulted in a number of highly impactful discoveries, ranging from novel gene and protein candidates, to diagnostic signatures obtained through neuroimaging, and real-time behavioral therapies delivered on Google Glass. As interdisciplinary research grows to become the rule rather than the exception, the ability to distinguish the various forms of autism from one another and provide personalized therapies can become the reality rather than the goal.

\section{Future perspective}

There are many forms of autism with behavioral signatures that overlap with a number of other disorders including attention deficit hyperactivity disorder, obsessive-compulsive disorder and intellectual disability. Though there has been considerable growth in the field of autism research as well as a shift away from compartmentalized research, personalized multidisciplinary approaches that seek to disentangle autism into its many forms must become the rule. Continuing to follow this growing paradigm shift in research will undoubtedly result in the ability to identify diagnostic biomarkers and targets for therapeutic intervention, which will both substantially decrease the age at which diagnosis is possible and allow for tailor-made treatments, drastically improving outcomes.

\section{Financial \& competing interests disclosure}

The authors have no relevant affiliations or financial involvement with any organization or entity with a financial interest in or financial conflict with the subject matter or materials discussed in the manuscript. This includes employment, consultancies, honoraria, stock ownership or opinions, expert testimony, grants or patents received or pending, or royalties.

No writing assistance was utilized in the production of this manuscript.

\section{Open access \\ This work is licensed under the Attribution-NonCommer- cial-NoDerivatives 4.0 Unported License. To view a copy of this license, visit http://creativecommons.org/licenses/by- nc-nd/4.0/}

\section{Executive summary}

\section{Growth surrounding autism}

- Autism-centered research efforts have increased $194 \%$ over the last 15 years, totaling more than 13,900 unique publications.

- Since the year 2000, over 9400 researchers have made significant and impactful contributions to this field, participating in almost 105,000 unique collaboration instances, $52 \%$ of which were found to be interdisciplinary connections.

- Discoveries resulting from interdisciplinary collaborations have been found to have a significantly higher average impact than discoveries from collaborations within a single discipline $(p<<0.05)$.

Impact of cross-disciplinary efforts

- Interdisciplinary collaborations have produced highly impactful discoveries, many of which have the potential to shed light on the complex etiology of autism and develop into clinically valuable biomarkers that are increasingly more personalized. 


\section{References}

Papers of special note have been highlighted as:

- of interest

1 Developmental Disabilities Monitoring Network Surveillance Year Principal I: prevalence of autism spectrum disorder among children aged 8 years - autism and developmental disabilities monitoring network, 11 sites, United States, 2010. MMWR Surveill. Summ. 63(Suppl. 2), 1-21 (2014).

2 Abrahams BS, Geschwind DH. Advances in autism genetics: on the threshold of a new neurobiology. Nat. Rev. Genet. 9(5), 341-355 (2008).

3 American Psychiatric Association, American Psychiatric Association. DSM-5 Task Force. Diagnostic and Statistical Manual of Mental Disorders: DSM-5 (5th). American Psychiatric Association, Washington DC, USA (2013)

4 Lee K, Brownstein JS, Mills RG, Kohane IS. Does collocation inform the impact of collaboration? PLoS ONE 5(12), e14279 (2010).

5 Duda M, Nelson T, Wall DP. Cross-pollination of research findings, although uncommon, may accelerate discovery of human disease genes. BMC Med. Genet. 13, 114 (2012).

- This analysis of cross-disease research found that the frequency of collaboration between genetically related disease fields was extremely low, but fields that participated in the most cross-disease collaboration had the highest rates of discovery.

6 Gibney G, Baxevanis AD. Searching NCBI Databases Using Entrez. Curr. Protoc. Hum. Genet. doi:10.1002/0471142905. hg0610s71 (2011) (Epub ahead of print).

7 Thomson Reuters. www.thomsonreuters.com/journal-citation-reports/

8 Corominas R, Yang X, Lin GN et al. Protein interaction network of alternatively spliced isoforms from brain links genetic risk factors for autism. Nat. Commun. 5, 3650 (2014).

9 Klei L, Sanders SJ, Murtha MT et al. Common genetic variants, acting additively, are a major source of risk for autism. Mol. Autism 3(1), 9 (2012).

- An innovative analysis focused on common genetic variants rather than rare de novo variants, which found that the additive effects of multiple common variants contribute up to $60 \%$ of total genetic autism spectrum disorders risk.

10 Gaugler T, Klei L, Sanders SJ et al. Most genetic risk for autism resides with common variation. Nat. Genet. 46(8), 881-885 (2014).

11 Samocha KE, Robinson EB, Sanders SJ et al. A framework for the interpretation of de novo mutation in human disease. Nat. Genet. 46(9), 944-950 (2014).

- This study details the development of a statistical model to better interpret the significance of de novo mutations. This model was then applied to a set of 1078 trios, with results suggesting a disruption of fundamental neurodevelopmental processes that can possibly be attributed to de novo mutations in autism.

12 Neale BM, Kou Y, Liu L et al. Patterns and rates of exonic de novo mutations in autism spectrum disorders. Nature 485(7397), 242-245 (2012).
13 Lim ET, Raychaudhuri S, Sanders SJ et al. Rare complete knockouts in humans: population distribution and significant role in autism spectrum disorders. Neuron 77(2), 235-242 (2013).

14 Yu TW, Chahrour MH, Coulter ME et al. Using wholeexome sequencing to identify inherited causes of autism. Neuron 77(2), 259-273 (2013).

15 O'roak BJ, Vives L, Girirajan S et al. Sporadic autism exomes reveal a highly interconnected protein network of de novo mutations. Nature 485(7397), 246-250 (2012).

16 Sanders SJ, Murtha MT, Gupta AR et al. De novo mutations revealed by whole-exome sequencing are strongly associated with autism. Nature 485(7397), 237-241 (2012).

17 Sebat J, Lakshmi B, Malhotra D et al. Strong association of de novo copy number mutations with autism. Science 316(5823), 445-449 (2007).

18 Rzhetsky A, Bagley SC, Wang K et al. Environmental and state-level regulatory factors affect the incidence of autism and intellectual disability. PLoS Comput. Biol. 10(3), e1003518 (2014).

19 Gotham K, Risi S, Pickles A, Lord C. The autism diagnostic observation schedule: revised algorithms for improved diagnostic validity. J. Autism Dev. Disord. 37(4), 613-627 (2007).

20 Lord C, Rutter M, Le Couteur A. Autism diagnostic interview-revised: a revised version of a diagnostic interview for caregivers of individuals with possible pervasive developmental disorders. J. Autism Dev. Disord. 24(5), 659-685 (1994).

21 Uddin LQ, Menon V, Young CB et al. Multivariate searchlight classification of structural magnetic resonance imaging in children and adolescents with autism. Biol. Psychiatry 70(9), 833-841 (2011).

22 Wolff JJ, Gu H, Gerig G et al. Differences in white matter fiber tract development present from 6 to 24 months in infants with autism. Am. J. Psychiatry 169(6), 589-600 (2012).

23 Bosl W, Tierney A, Tager-Flusberg H, Nelson C. EEG complexity as a biomarker for autism spectrum disorder risk. BMC Med. 9, 18 (2011).

- This study provides support for the use of EEG signals as an affordable and noninvasive method for the early, accurate detection of autism risk.

24 Di Martino A, Yan CG, Li Q et al. The autism brain imaging data exchange: towards a large-scale evaluation of the intrinsic brain architecture in autism. Mol. Psychiatry 19(6), 659-667 (2014).

25 Skafidas E, Testa R, Zantomio D, Chana G, Everall IP, Pantelis C. Predicting the diagnosis of autism spectrum disorder using gene pathway analysis. Mol. Psychiatry 19(4), 504-510 (2014).

26 Glatt SJ, Tsuang MT, Winn M et al. Blood-based gene expression signatures of infants and toddlers with autism. J. Am. Acad. Child Adolesc. Psychiatry 51(9), 934-944 e932 (2012).

27 Kong SW, Collins CD, Shimizu-Motohashi Y et al. Characteristics and predictive value of blood transcriptome 
signature in males with autism spectrum disorders. PLoS ONE 7(12), e49475 (2012).

- An extensive blood transcriptome study which developed a 55 -gene prediction model that performed with $73 \%$ classification accuracy in males, showing promise for use as a blood expression profiling method for detecting autism.

28 Synapdx.

www.synapdx.com/clinical_study.html

29 Wall DP, Dally R, Luyster R, Jung JY, Deluca TF. Use of artificial intelligence to shorten the behavioral diagnosis of autism. PLoS ONE 7(8), e43855 (2012).

30 Wall DP, Kosmicki J, Deluca TF, Harstad E, Fusaro VA. Use of machine learning to shorten observation-based screening and diagnosis of autism. Transl. Psychiatry 2, e100 (2012).

31 Duda M, Kosmicki JA, Wall DP. Testing the accuracy of an observation-based classifier for rapid detection of autism risk. Transl. Psychiatry 4, e424 (2014).

32 Fusaro VA, Daniels J, Duda M et al. The potential of accelerating early detection of autism through content analysis of YouTube videos. PLoS ONE 9(4), e93533 (2014).

- A proof-of-principle study that validates the use of short unstructured home videos for capturing behaviors used to clinically assess autism via the Autism Diagnostic Observation Schedule, as well as the use of nonclinical raters to accurately code the behaviors.
33 Lovaas OI. Behavioral treatment and normal educational and intellectual functioning in young autistic children. J. Consult. Clin. Psychol. 55(1), 3-9 (1987).

34 Koegel LK, Koegel RL, Harrower JK, Carter CM. Pivotal response intervention I: overview of approach. J. Assoc. Pers. Severe. 24(3), 174-185 (1999).

35 Koegel LK, Koegel RL, Shoshan Y, Mcnerney E. Pivotal response intervention II: preliminary long-term outcome data. J. Assoc. Pers. Sever. 24(3), 186-198 (1999).

36 Vismara LA, Rogers SJ. The early start denver model a case study of an innovative practice. J. Early Intervention 31(1), 91-108 (2008).

37 Austism Speaks. www.autismspeaks.org/autism-apps

38 Autism therapy on glass. www.wall-lab.stanford.edu/projects

39 Faja S, Webb SJ, Jones E et al. The effects of face expertise training on the behavioral performance and brain activity of adults with high functioning autism spectrum disorders. J. Autism Dev. Disord. 42(2), 278-293 (2012).

40 Frye RE. Clinical potential, safety, and tolerability of arbaclofen in the treatment of autism spectrum disorder. Drug Healthc. Patient Saf. 6, 69-76 (2014).

41 Siniscalco D, Bradstreet JJ, Sych N, Antonucci N. Mesenchymal stem cells in treating autism: novel insights. World J. Stem Cells 6(2), 173-178 (2014). 